\title{
Combining TrkA Immunohistochemical-Score with Clinicopathologic Parameters in Neuroblastoma: An Influential Prognostic Nomogram
}

\section{Juanqing Yue}

Shanghai Jiaotong University School of Medicine Xinhua Hospital

\section{Lei Cai}

Shanghai Jiaotong University School of Medicine Xinhua Hospital

\section{Ruifen Wang}

Shanghai Jiaotong University School of Medicine Xinhua Hospital

\section{Meng Qiao}

Shanghai Jiaotong University School of Medicine Xinhua Hospital

\section{Kezhou Wang}

Shanghai Jiaotong University School of Medicine Xinhua Hospital

\section{Wenwei Yu}

Shanghai Jiaotong University School of Medicine Xinhua Hospital

Junlei Li

Shanghai Jiaotong University School of Medicine Xinhua Hospital

\section{Meng Zhang}

Shanghai Jiaotong University School of Medicine Xinhua Hospital

\section{Wenbin Guan}

Shanghai Jiaotong University School of Medicine Xinhua Hospital

lifeng Wang ( $\nabla$ wanglifeng@xinhuamed.com.cn)

Shanghai Jiaotong University School of Medicine Xinhua Hospital https://orcid.org/0000-0002-71180163

\section{Primary research}

Keywords: neuroblastoma, NTRK1, TrkA, immunohistochemistry, bioinformatics analysis, prognosis, Trk inhibitors, nomogram

Posted Date: January 14th, 2021

DOI: https://doi.org/10.21203/rs.3.rs-143769/v1 
License: (c) (i) This work is licensed under a Creative Commons Attribution 4.0 International License. Read Full License 
1 Title Page

2 Combining TrkA Immunohistochemical-score with

3 Clinicopathologic parameters in Neuroblastoma: An Influential

$4 \quad$ Prognostic Nomogram

5 - Authors' names: Juanqing Yue†, Lei Cai†, Ruifen Wang, Meng Qiao, Kezhou

6 Wang, Wenwei Yu, Junlei Li, Zhang Meng, Wenbin Guan*, Lifeng Wang*

7 - Authors'affiliations: Department of Pathology, Xinhua Hospital Affiliated to

8 Shanghai Jiao Tong University School of Medicine, Shanghai, China

9 * Correspondence:

10 Wenbin Guan

11 guanwenbin@xinhuamed.com.

12 Lifeng Wang

13 wanglifeng@xinhuamed.com.

Abstract:

【Background】Neuroblastoma (NB) is one of the most common solid tumors in children with varied clinical outcomes. Although there are some several risk stratification systems currently, their clinical applications are limited due to the testing conditions of different laboratory and the heavy financial burden on patients. TrkA is coded by NTRK1, belonging to tropomyosin receptor kinase family. We have observed that TrkA was differentially expressed in paraffin tissue sections of NB. The aim of this 
study was to determine the immunohistochemical-score of TrkA as an independent prognostic factor for $\mathrm{NB}$ and establish a useful prognostic model for postoperative patients.

【 Methods 】 We systematically summarized the relationship between immunochemistry (IHC) score of TrkA and clinicopathological parameters in $86 \mathrm{NB}$ cases. Fluorescence in situ hybridization (FISH) and qRT-PCR were used to detect NTRK1 gene fusion. Furthermore, GSE96631, GSE16476, GSE49710 and GSE73537 datasets, originated from Gene Expression Omnibus (GEO), were analyzed to figure out the NTRKI related molecular characteristics by bioinformatics methods. And combined TrkA immunohistochemical-score with clinicopathologic parameters to construct a prognostic nomogram of overall survival (OS) for NB.

【Result】 In clinical samples and GEO database analyses, patients in the NTRK1 / TrkA low expression group showed significantly poorer outcome than patients in high group. Multivariate cox regression analysis demonstrated NTRK1 / TrkA as an independent prognostic factor for NB survival. Neither Fluorescence in situ hybridization nor qRT-PCR detected evidence for NTRKl gene fusion in clinical samples, indicating that differential expression in NTRK1 / TrkA are caused by epigenetic changes. Bioinformatics analyses revealed that $M Y C$ target related pathway may play a critical role in low expression of TrkA, leading to unfavorable prognoses of NB. 
【Conclusion】 The results of this study suggest that the IHC score of TrkA may be used as an independent predictor of postoperative OS of patients with NB. By combining the IHC score of TrkA and clinicopathological features, the proposed nomogram provides a feasible predictive tool for postoperative patients with NB. Simultaneously, this study also reveals that Trk inhibitors are not supposed to be taken in NB patients.

Keywords: neuroblastoma, NTRK1, TrkA, immunohistochemistry, bioinformatics analysis, prognosis, Trk inhibitors, nomogram

\section{Number of Figures: 7}

\section{Number of Tables: 5}

\section{Number of Supporting Information:9}

\section{Introduction}

In 1864, neuroblastoma (NB) was first described by Rudolf Virchow as a glioma tumor occurring in the abdominal cavity of children. Then it was named by James Homer Wright in 1910 as neurocytoma or neuroblastoma[1]. NB is one of the malignant solid tumors that often occur in children, with a median age of 18 months. Although typical lesions can also be seen in adults, these cases are extremely rare[2].

NB is a sympathetic nervous system embryogenic tumor originating from primitive neural crest cells, which can occur anywhere in the 
sympathetic nervous system[3]. NB belongs to the neuroblastic tumor (NT) spectrum. According to different differentiation levels, NTs are categorized as ganglioneuroma $(\mathrm{GN})$, intermediate ganglioneuroblastoma (GNB), and neuroblastoma (NB)[4]. NB is a type of complex disease showing different clinical, biological and genetic characteristics. Some patients may have spontaneous regression, while others may have disease progression or even death. Because of the trait of invasiveness and metastasis, NB accounts for nearly $15 \%$ of all childhood malignant tumor deaths[5]. Current risk stratification revealed that the age of initial diagnosis and the status of $M Y C N$ gene are the most powerful prognostic factor. Older age at initial diagnosis and $M Y C N$ amplification imply unfavorable outcome. Besides, many other factors matter, such as chromosomal deletion or acquisition[6], and DNA ploidy[7]. At present, there are mainly two kinds of risk classification systems in the world: COG (Children's Oncology Group ) classification system[8] and INRG (International Neuroblastoma Risk Group) classification system $[9,10]$. The former introduced five risk factors to divide NB into three groups: low risk, intermediate risk and high risk. The five risk factors included INSS stage, age of initial diagnosis, $M Y C N$ amplification status, international pathological classification of NB and DNA ploidy. The INRG classification system divided NB into four groups: very low risk, low risk, intermediate risk and high risk. It took seven risk factors into consideration: 
INRG stage, age, MYCN amplification status, international pathological classification of NB, tumor cell differentiation, DNA ploidy and chromosome $11 \mathrm{q}$ abnormality. However, their clinical applications are limited due to the limitations of different laboratory testing conditions and complexity of operation. As for some patients who cannot get available test, he systematic prediction of the risk of progrnosis is a formidable task. Therefore, it is necessary to develop new effective and convenient models related to prognosis of NB contributing to stratified risk management of patients and further appropriate therapeutic strategies.

Neurotrophic receptor tyrosine kinase (NTRK) gene family includes NTRK1, $N T R K 2$ or $N T R K 3$, coding for three transmembrane high-affinity tyrosine-kinase receptors (TrkA, TrkB, and TrkC) for nerve growth factors respectively, which is mainly involved in nervous system development[11,12]. Current studies have shown that NTRK was activated through multiple mechanisms in various malignant tumors, but $N T R K$ fusion is the most definite driving factor for carcinogenesis and can cause overexpression of Trk protein. There are some approved Trk small molecule inhibitors, such as Entrectinib and Larotrectinib, can be used in patients with advanced solid tumors carrying NTRK fusion genes. Recently, some studies have found high expression of NGF/TrkA accompanied with malignant biological behavior in pancreatic cancer and breast cancer[13-15]. 

in NB patients, but its clinical significance is not clear yet. Previous

111 studies have found that the mRNA expression level of NTRK1 was related

112 to the poor prognosis of NB, high expression of mRNA for NTRK1 tending 113 to be with favorable progression of $\mathrm{NB}[16-19]$. But there were few 114 researches to verify the relationship between the expression level of TrkA, 115 the protein product of NTRK1 gene, and clinical features among NB's 116 patients. In addition, the mechanisms of NTRK1 differential expression 117 level causing different clinical outcomes are still indefinite yet.

118 In this study, we combined clinical samples and public microarray 119 datasets, and concentrated on exploring potential feasibility of TrkA 120 immunohistochemical-score as an evaluation model for prediction of 121 outcome in NB patients, aiming to provide a basis for prognostic and 122 therapeutic guidance. Moreover, bioinformatics methods were used to 123 analyze the possible mechanisms behind.

\section{Material and Methods}

\subsection{Specimen Collection}

The 86 clinical FFPE tissue samples diagnosed as NTs in this study

127 were collected by the Department of Pathology of Xinhua Hospital Affiliated To Shanghai Jiao Tong University School Of Medicine from 2013 to 2020, including 52 cases of NB, 29 cases of GNB and 5 cases of

GN, and information about patient's basic clinicopathological characteristics is 
131

132

133 published standardizations of diagnosis, and classified based on the

134 International Neuroblastoma Staging System (INSS). Each specimen

135 collected was consented to by both hospital and individual. This study was

136 approved by the Medical Ethical Committee of Xinhua Hospital Affiliated

137 to Shanghai Jiao Tong University School of Medicine.

shown in Table 1. All patients underwent surgery in the hospital. Patients were diagnosed by pathologists according to World Health Organization

\subsection{Microarry source}

In this study, we downloaded the gene expression profiling data from the GEO database (https://www.ncbi.nlm.nih.gov/geo/). A total of 311 series about human NB were retrieved from the database. Finally, we select four gene expression profiles (GSE73517, GSE16476, GSE49710 and GSE96631) with deliberation (Table 2), and detailed information is shown in Tables S1-S4.

2.3 Immunohistochemistry (IHC)

IHC staining was performed with an Anti-bodies against TrkA (EPR17341, Abcam, the United Kingdom) on an automated system (BOND MAX system, Leica, Germany) following operating instructions. A positive result is defined as staining of the cytoplasm, and/or cell membrane and/or nucleus. Inflammatory cells were viewed as internal controls. 

quantified by using the semiquantitative integration method based on the

154 staining intensity and the positive rate (Figure 1a). Detailly, the scoring 155 criterion for staining intensity was following: compared with the 156 background color, colorless was 0 point, light yellow, yellow-brown and 157 brown were counted as 1, 2, and 3 points respectively. The staining 158 intensity was compared with the background color. The scoring standard 159 for percentage of positive cells were following: <25\%, 25\% 50\%, $51 \sim 75 \%,>75 \%$ were scored as $1,2,3,4$ points respectively. The final 161 score $=$ staining intensity score $\times$ positive rate score.

2.4 Fluorescence in situ hybridization (FISH) and qRT-PCR

FISH can detect large structural variants at the DNA level, such as abnormalities in the number and structure of chromosomes or genes. The MYCN copy number was identified by FISH (Vysis LSI $N$-MYC CEP 2 spectrum orange, Abbott, the United States). MYCN was considered to be amplified when the gene copy number was above 3 per haploid. All clinical cases were tested $M Y C N$ status. In this study, NTRKI fusion was detected by separation probe (NTRK1 Break Apart Rearrangement Probe, F.01103. An Biping, China) and was only performed on the cases with strongly IHC staining $(n=22)$. However, there is no clear standard for the positive

172 threshold currently. In our study, of the 50 cells evaluated, more than $30 \%$ 173 tumor cells detected separate signals, was viewed as fusion status of 
174 175 pathologists independently. for target gene.

the NTRK1. The interpretation of the above results was assessed by two

qRT-PCR was performed to further verify NTRK1 fusion. We selected NB samples from the above 22 cases which met the following testing conditions: 1) samples need to be obtained in recent two years; 2) the content of tumor cells in the tissue should exceed $30 \%$. Total RNA extraction from FFPE specimens was performed using the kit (8.126001W008A, Amoy Dx, China) according to the manufacturer's instructions. The RNA of the tumor cells in the FFPE samples were extracted and reverse transcribed into cDNA, then combined with fluorescent probe technology to test the $N T R K$ gene fusion qualitatively on a fluorescent quantitative PCR instrument (ABI 7500, Thermo Fisher, the United States). When the CT (cycle times) value of HEX (VIC) signal for housekeeping gene is $<20$, and simultaneously CT value of FAM signal for target gene is $\leq 25$, the sample was thought to be detected as gene fusion

\subsection{Bioinformatics analysis of microarray datasets}

To explore the function of NTRK1 in the prognosis of NB, all NB cases in three datasets (GSE73517, GSE16476 and GSE49710) were divided into $N T R K 1$ high-expression group (NB-NTRK $\left.1^{\text {high }}\right)$ and $N T R K 1$ low-expression group (NB-NTRK $1^{\text {low }}$ ) respectively according to the the median value of NTRK1 expression level. The Sanger box software was used to detect the 
differentially expressed genes (DEGs) between two groups, and the

197 adjusted P-value and $|\log F C|$ were calculated. When genes met the cutoff 198 criteria, adjusted $\mathrm{P}<0.05$ and $|\log \mathrm{FC}| \geq 1.0$, those genes were considered as 199 DEGs. Making a statistical analysis of each dataset and use the Venn 200 diagram webtool (bioinformatics.psb.ugent.be/webtools/Venn/) to 201 determine the intersecting part. Then Gene Ontology (GO) functional 202 annotation analysis is a common strategy for extensive functional 203 enrichment studies, which is divided into biological process (BP), cellular components (CC) and molecular functions (MF). Using the Database for Annotation, Visualization and Integrated Discovery (DAVID) tools 206 (https://david.ncifcrf.gov/), we can achieve GO annotation analysis of 207 DEGs. $\mathrm{P}<0.05$ and gene counts $\geq 10$ were considered statistically 208 significant. Then we visualized the protein-protein interaction (PPI) 209 network of DEGs by using Cytoscape software (www.cytoscape.org/). 210 Finally, Gene Set Variation Analysis (GSVA) was carried out in 211 NB-NTRK $1^{\text {high }}$ and NB-NTRK $1^{\text {low }}$ groups by the GSVA R package that also 212 annotated gene set c2.cp.kegg.v7.0.symbols.gmt. Then the results of GSVA 213 were intersected by $\mathrm{R}$ package 214 ( http://cran.r-project.org/web/packages/VennDiagram/), and the venn 215 diagram was drawn. 
was based on the results of the multivariate regression analysis, combining with pathological diagnosis, and compiled using the RMS package in R software. Student's two-tailed t test, Chi-square test and survival analysis were used in statistical analysis. When $\mathrm{P}$ value $<0.05$ was considered statistically

223 significant.

\section{Results}

3.1 The correlation between NTRK1/ TrkA and NB clinical parameters

TrkA protein expression levels were assessed in $86 \mathrm{NB}$ clinical samples using IHC. TrkA protein were differentially expressed between clinical samples (Figure 1b-c). Moreover, correlation of IHC score of TrkA and clinical parameters were found out that IHC score of TrkA was strongly associated with age of initial diagnosis ( $\leq 18$ months/ $>18$ months), MYCN status (amplification or not) and INSS stage. The results turn out that IHC score of TrkA trended to be higher in younger, $M Y C N$ not amplified, and low INSS stage (stage 1 and 2) or 4s patients. There was no significant difference in gender. Further, we compared the expression of TrkA score in the primary tumor and the metastasis in 32 of 86 clinical cases which the FFPE tissue of metastases can be obtained. It was found that there was no statistical difference in the IHC score of TrkA of tumor cells between metastasis and primary tissue (Figure 1d, $\mathrm{P}=0.5526$ ). 
The microarray datasets (GSE73517, $\mathrm{n}=105 ;$ GSE16476, $\mathrm{n}=88$; GSE49710, $n=498$ ) were obtained to further verify the relationship

241 between mRNA expression level of NTRK1 and clinical characteristics of

242 NB. The mRNA expression level of NTRK1 was higher in younger, MYCN 243 not amplified and low INSS stage (stage 1 and 2) or 4 s stage, but did not 244 significantly correlate with gender $(\mathrm{P}>0.05)$ (Figure 2a-d), which was 245 consistent with analyses derived from clinical samples. The result concluded from GSE96631 showed significant downregulated mRNA expression of NTRK1 in NB patients compared with embryonic neural creat cells (ENCC)、 adrenal cortex (AC) and adrenal marrow (AM) $(\mathrm{P}<0.0001$,

249 Figure 2e).

According to the the median value level of NTRK1, all NB cases in three datasets were divided into NB-NTRK $1^{\text {high }}$ groups and NB-NTRK $1^{\text {low }}$ groups respectively. The correlation analysis results (Table 3 ) revealed that the

253 NTRK1 expression level in NB patients significantly correlated with age of 254 initial diagnosis, MYCN status and INSS stage $(\mathrm{P}<0.05)$.

\subsection{NTRK $1^{\text {low }}$ is associated with unfavorable outcome in NB patients}

According to the IHC score and the median value (score=6) as the 257 cut-off value, the 86 clinical samples were divided into high-expression 258 (Trk $\left.\mathrm{A}^{\text {high }}\right)$ and low-expression $\left(\right.$ TrkA $\left.^{\text {low }}\right)$ groups, and survival analyses 259 were carried out. Besides, the two datasets (GSE16476 and GSE49710) 260 which prognostic information were available were also analyzed for 
survival. The result derived from clinical cohort showed

262 that TrkA $^{\text {low }}$ group was significantly associated with shorter overall

263 survival (OS) time, as well as the result got from public datasets, showing

264 lower mRNA expression of NTRK1 indicating shorter OS time (Figure 3a).

265 In the multivariate Cox regression analysis of OS, the variables included were age of 266 first diagnosis, sex, INSS stage, $M Y C N$ status and the IHC score of TrkA or mRNA 267 expression level of NTRK1 (Figure 3b-d). Through 86 clinical sample 268 analysis, it presented that the prognosis of NB had a strong correlation with the TrkA score and the status of MYCN gene. In both GSE49710 and GSE16476, the prognosis was associated with age and NTRK1 expression 271 level, besides, in GSE49710, the results identified that the prognosis of NB 272 patients is also significantly correlated with INSS stage and $M Y C N$ status

In order to assess the specificity and sensitivity of $N T R K 1 / \operatorname{TrkA}$ as a 274 reliable predictive factor of OS, Receiver Operator Characteristic curve 275 (ROC), Decision Curve Analysis (DCA) analyses and nomogram were 276 further performed. The AUCs of the TrkA among clinical samples, 277 GSE16476 and GSE49710 were $0.784,0.878$ and 0.815 , respectively. DCA 278 suggesting that TrkA has good clinical utility for prognostic evaluation 279 (Figure 3e-f). Based on the results of the multivariate analysis of OS, combining 280 pathological diagnosis, we established a prognostic nomogram of OS (Figure 4). Data 281 analysis showed that TrkA score0 better than 3 in the nomogram, this may be due to 282 the cases of score $0(n=4)$ and $3(n=1)$ is limited. Besides, score 4 ranks between 6 and 8 
283

284

285

286

287

288

289

290

291

292

293

294

295

296

297 (Figure 5c).

in the nomogram may owing to insufficient follow-up time of some cases because the time of inclusion of cases is different. However, it showed the trend that OS was significantly increased with the increase in scores, the C-index $(=0.95)$ manifested the preferable effectiveness of model.

\subsection{FISH and qRT-PCR}

FISH techniques were performed in FFPE samples (Figure 5a). No fusion status of NTRK1 was found in above cases. PCR amplification curve of positive control showed that the cycle threshold (CT) value of HEX (VIC) signal for housekeeping gene is $12.5(\leq 20)$, and CT value of FAM signal for target gene is $13.43(\leq 25)$, prompting the experimental conditions are qualified (Figure 5b). PCR amplification curve of clinical sample showed that the CT value of HEX (VIC) signal for housekeeping gene is 12.27 $(\leq 20)$, and CT value of FAM signal for target gene is undetermined, implying that no evidence involving NTRKI fusion was detected by qRT-PCR

\subsection{GO Functional enrichment analysis and GSVA analysis}

To probe the function of NTRKl in the good prognosis of NB, we conducted a bioinformatics analysis on the database data. With the criteria of FDR $<$ 0.05 and $|\log \mathrm{FC}| \geq 1$, total of 1978 DEGs were identified from GSE73517, including 1839 upregulated genes and 139 downregulated genes, while 407 DEGs were identified from GSE16476, 210 upregulated genes and 197 downregulated genes. And 833 DEGs were identified in GSE49710, 
including 769 genes were upregulated, and 64 genes were downregulated

(Table 4 and Figure 6a). All DEGs were selected out through comparing NB-NTRK $1^{\text {high }}$ and NB-NTRK $1^{\text {low }}$ samples. Detailed information about DEGs of each data set were included in Tables S5-S7. Subsequently, Venn analysis was performed to get the intersection of the DEGs profiles (Figure 6b). Finally, 151 DEGs were significantly differentially expressed among all three groups, including 129 upregulated genes and 22 downregulated genes, and a summary of intersection of the DEGs profiles is displayed in Tables S8. GO function for DEGs were performed using the DAVID (Table 5). The results of GO analysis indicated that DEGs were mainly enriched in BPs, including positive regulation of GTPase activity and apoptotic process. CC analysis showed that the DEGs were significantly enriched in plasma membrane and integral component of plasma membrane. As for MF analysis, the DEGs were enriched in calcium ion binding. Details about enriched GO terms of DEGs is shown in Tables S9. Protein interactions among the DEGs were predicted with STRING tools. Totally, 149 nodes and 135 edges were involved in the PPI network (Figure 6c).

The function of NTRK1 was further revealed by GSVA by comparing the differential expression group (Figure 7). The most significant HALLMARK gene set in the NTRK1 low expression group was MYC targets, which was the intersected part of venn diagram for the GSVA analysis results of the three data sets. On the contrary, in the NTRK1 high 
expression group, the common HALLMARK gene set was hedgehog (Hh) signaling pathway.

\section{Discussion}

NB is one of the most common solid tumors in children, with highly variable symptoms and clinical process. Although some risk stratification strategies are available, their clinical applications remain limited owing to their complexity of operation and expensive testing costs.

There are many techniques in pathology, the immunohistochemistry technology has become a mature technology after more than 40 years of development. In the past 20 years, it has been widely used in medical biology research and diagnostic pathology practice. It has become an important method to identify antigen molecules such as proteins, peptides and carbohydrates in tissues and cells. Compared with complex and expensive molecular tests, immunohistochemical staining techniques are faster and more economical, moreover, it consumes less tissue. Most pathology laboratories of the hospital have launched immunohistochemical testing projects, which makes this test be available to nearly all patients.

This study is the first time that TrkA has been quantitative evaluated by immunohistochemical staining in a large sample of neuroblastoma and constructed a prognostic nomogram of OS for NB. IHC score of TrkA is easy to conduct that can be substituted for texting mRNA expression level of 
NTRK1. Patients with higher IHC score of TrkA tend to have better prognoses.

FISH and qRT-PCR were used to detect the NTRK1 gene status in the TrkA overexpression samples, but no fusion was found. Furthermore, bioinformatics analyses revealed that $M Y C$ targets was the obvious pathway that highly associated with the low expression of NTRK1/TrkA, taking unignorable effects in poor prognoses of NB. The $M Y C(c-M Y C)$ gene, along with the $M Y C N$ gene, belongs to the $M Y C$ gene family. Their encoding protein products, either MYCN protein or MYC protein, are known to make a complex with MAX (MYC-associated factor X) protein, and the complex binds to target genes, leading to activation of transcription, involving in cell proliferation, inhibition of differentiation, apoptosis, and so on[20]. Recent studies proposed that $M Y C$ family-driven NB, defined as augmented expression of MYC protein and/or MYCN protein, is highly aggressive. And MYC protein overexpression has been reported as a novel prognostic factor for NB[21-24]. Based on previous studies and our results, TrkA may promote the cell proliferation, migration and invasion of neuroblastoma through the $M Y C$ axis.

Contrastively, the Hh signaling pathway was the associated with the high expression of NTRK1. Many studies have noticed the activating effect of Hh signaling on autophagy. For instance, Hh antagonist cyclopamine inhibited autophagy activation in NB cell line SHSY5Y, suggesting that Hh signaling mediated the activation of autophagy in NB cell lines. Besides, some researchers observed that 
in cell line $\mathrm{C} 3 \mathrm{H} 10 \mathrm{~T} 1 / 2$, suppression of $\mathrm{Hh}$ signaling activation led to decreased protein expression of ATG5, a key protein for autophagy activation[25]. Moreover, some researchers believed that the $\mathrm{Hh}$ signaling pathway may promote differentiation of NB. Above researches uncovered a probable explanation for spontaneous regression of NB. In the light of these, we could conceive that the activation of the Hh signaling pathway in NB is dramatically connected with the high expression of $N T R K 1$, which may play a crucial role in good prognosis of NB, that was consistent with previous study[26,27]. Based on previous research, we can know that the downstream pathways of NTRK gene are PI3K, RAS/MAPK/ERK and PLC- $\gamma$ pathway[28], which are independent of either $M Y C$ targets or Hh signaling pathways. Therefore, the above results mean that the different expression of TrkA among NB patients was caused by epigenetic changes, instead of gene alterations.

$$
\text { Besides, analyzing about IHC score of TrkA between metastases and }
$$
primary tumors among clinical samples, no significant statistical difference was found. Therefore, when the primary tumors were difficult to be obtained clinically, metastases were supposed to be detected.

There are some highlights of our study. Firstly, we constructed a model based on a biomarker associated with outcomes of NB. Besides, the technology was easy to obtained by most laboratories and to some extent can relieve financial pressure of patient's family. Secondly, the results of pathological molecular testing suggest that NTRK1 gene fusion was not 
392 found in NB, and TrkA high expression may contribute a good outcome, so

393 the usage of Trk inhibitors maybe inadequate for NB. Thirdly,

394 bioinformatic analysis were performed to explore underlaying mechanism, 395 providing a novel view for NB.

\section{5. Conclusion}

397 In this study, we proposed a useful predictor model easy to operate 398 technically. The IHC score of TrkA in tumor cells can be used as a 399 supplementary material, contributing to individualized treatment and 400 optimizing the hierarchical management of children with NB.

\section{6.Abbreviations}

402 CI: Confidence interval

403 DEGs: Differentially expressed genes

404 GSEA: Gene set enrichment analysis

405 FFPE: Formalin-fixed and paraffin-embedded

406 FISH: Fluorescence in situ hybridization

407 GEO: Gene Expression Omnibus

408 IHC: Immunohistochemical

409 TrkA: Tyrosine kinase A

410 NB: neuroblastoma

$411 \quad$ NTRK 1: neurotrophic receptor tyrosine kinase 1

412 PPI: Protein-protein interaction

413 ROC: Receiver operating characteristic 
7.Declarations

7.1 Ethics approval and consent to participate

Each specimen collected was consented to by both the Medical Ethical

School of Medicine and individual and every patient's parents.

The authors consent for publication.

7.3 Availability of data and materials

The datasets analyzed in this study were obtained from Gene Expression

425 Omnibus (GEO) database for free. The accession numbers are mentioned in

GSE49710).

\subsection{Competing interests}

429 The authors declare that they have no competing interests.

\subsection{Funding}

431 This study is sponsored by the Joined Medicine-Engineering Fund of 432 Shanghai Jiao Tong University (No. YG2016QN71).

\subsection{Author' Contributions}

434 JY and LC performed the research study, analyzed the data, and wrote 435 the paper. RW, MQ, KW, and WY supported during performing of the 
436

437

experiments, and contributed essential reagents and laboratory equipment.

JL and ZM collected patient data. WG and LW conceived and designed the study. All authors contributed to the article and approved the submitted version.

$$
\text { Corresponding author }
$$

$$
\text { Correspondence to Wenbin Guan \& Lifeng Wang }
$$

\subsection{Acknowledgments}

None.

\subsection{Authors' information}

Juanqing Yue and Lei Cai contributed equally to this work.

Affiliations: Department of Pathology, Xinhua Hospital Affiliated to Shanghai Jiao

\section{Tong University School of Medicine, Shanghai, China}

Juanqing Yue, Lei Cai, Ruifen Wang, Meng Qiao, Kezhou Wang, Wenwei Yu, Junlei Li, Zhang Meng, Wenbin Guan \& Lifeng Wang

\section{References:}

1. Wright JH. Neurocytoma or neuroblastoma, a kind of tumor not generally recognized. The Journal of experimental medicine. 1910; 12: 556-61.

2. Suzuki M, Kushner BH, Kramer K, Basu EM, Roberts SS, Hammond WJ, LaQuaglia MP, Wolden SL, Cheung N-KV, Modak S. Treatment and outcome of adult-onset neuroblastoma. International journal of cancer. 2018; 143: 1249-58.

3. Nakagawara A, Li Y, Izumi H, Muramori K, Inada H, Nishi M. Neuroblastoma. Japanese 
459 4. Xie Y, Xu H, Fang F, Li Z, Zhou H, Pan J, Guo W, Zhu X, Wang J, Wu Y. A 3-Protein Expression Signature of Neuroblastoma for Outcome Prediction. Am J Surg Pathol. 2018; 42: 1027-35.

5. Ward E, DeSantis C, Robbins A, Kohler B, Jemal A. Childhood and adolescent cancer statistics, 2014. CA: a cancer journal for clinicians. 2014; 64: 83-103.

8. Strother DR, London WB, Schmidt ML, Brodeur GM, Shimada H, Thorner P, Collins MH, Tagge E, Adkins S, Reynolds CP, Murray K, Lavey RS, Matthay KK, Castleberry R, Maris JM, Cohn SL. Outcome after surgery alone or with restricted use of chemotherapy for patients with low-risk neuroblastoma: results of Children's Oncology Group study P9641. Journal of clinical oncology : official journal of the American Society of Clinical Oncology. 2012; 30: 1842-8.

9. Maris JM. Recent advances in neuroblastoma. N Engl J Med. 2010; 362: 2202-11.

10. Cohn SL, Pearson AD, London WB, Monclair T, Ambros PF, Brodeur GM, Faldum A, Hero B, lehara T, Machin D, Mosseri V, Simon T, Garaventa A, Castel V, Matthay KK. The International Neuroblastoma Risk Group (INRG) classification system: an INRG Task Force report. Journal of clinical oncology : official journal of the American Society of Clinical 
11. Nakagawara A. Trk receptor tyrosine kinases: A bridge between cancer and neural development. Cancer Letters. 2001; 169: 107-14. L, Sartore-Bianchi A, Vanzulli A, Cresta S, Damian S, Duca M, Ardini E, Li G, Christiansen J, Kowalski K, Johnson AD, Patel R, Luo D, Chow-Maneval E, Hornby Z, Multani PS, Shaw AT, De Braud FG. Safety and Antitumor Activity of the Multitargeted Pan-TRK, ROS1, and ALK Inhibitor Entrectinib: Combined Results from Two Phase I Trials (ALKA-372-001 and STARTRK-1). Cancer Discov. 2017; 7: 400-9.

13. Marchesi F, Piemonti L, Mantovani A, Allavena P. Molecular mechanisms of perineural invasion, a forgotten pathway of dissemination and metastasis. Cytokine \& growth factor reviews. 2010; 21: 77-82.

14. Liebig C, Ayala G, Wilks JA, Berger DH, Albo D. Perineural invasion in cancer: a review of the literature. Cancer. 2009; 115: 3379-91.

15. Romon R, Adriaenssens E, Lagadec C, Germain E, Hondermarck H, Le Bourhis X. Nerve growth factor promotes breast cancer angiogenesis by activating multiple pathways. Mol Cancer. 2010; 9: 157-.

16. Suzuki T, Bogenmann E, Shimada H, Stram D, Seeger RC. Lack of high-affinity nerve growth factor receptors in aggressive neuroblastomas. J Natl Cancer Inst. 1993; 85: 377-84.

Kogner P, Barbany G, Dominici C, Castello MA, Raschellá G, Persson H. Coexpression of messenger RNA for TRK protooncogene and low affinity nerve growth factor receptor in 
neuroblastoma with favorable prognosis. Cancer Res. 1993; 53: 2044-50.

18. Borrello MG, Bongarzone I, Pierotti MA, Luksch R, Gasparini M, Collini P, Pilotti S, Rizzetti MG, Mondellini P, De Bernardi B, et al. trk and ret proto-oncogene expression in human neuroblastoma specimens: high frequency of trk expression in non-advanced stages. Int J Cancer. $1993 ;$ 54: 540-5.

19. Nakagawara A, Arima-Nakagawara M, Scavarda NJ, Azar CG, Cantor AB, Brodeur GM. Association between high levels of expression of the TRK gene and favorable outcome in human neuroblastoma. N Engl J Med. 1993; 328: 847-54.

20. Meyer N, Penn LZ. Reflecting on 25 years with MYC. Nature reviews Cancer. 2008; 8: 976-90.

21. Ikegaki N, Shimada H, Fox AM, Regan PL, Jacobs JR, Hicks SL, Rappaport EF, Tang XX. Transient treatment with epigenetic modifiers yields stable neuroblastoma stem cells resembling aggressive large-cell neuroblastomas. Proceedings of the National Academy of Sciences of the United States of America. 2013; 110: 6097-102.

22. Tornóczky T, Kálmán E, Kajtár PG, Nyári T, Pearson AD, Tweddle DA, Board J, Shimada H. Large cell neuroblastoma: a distinct phenotype of neuroblastoma with aggressive clinical behavior. Cancer. 2004; 100: 390-7.

23. Wang LL, Teshiba R, Ikegaki N, Tang XX, Naranjo A, London WB, Hogarty MD, Gastier-Foster JM, Look AT, Park JR, Maris JM, Cohn SL, Seeger RC, Asgharzadeh S, Shimada H. Augmented expression of MYC and/or MYCN protein defines highly aggressive MYC-driven neuroblastoma: a Children's Oncology Group study. Br J Cancer. 2015; 113: 57-63.

24. Wang LL, Suganuma R, Ikegaki N, Tang X, Naranjo A, McGrady P, London WB, Hogarty MD, Gastier-Foster JM, Look AT, Park JR, Maris JM, Cohn SL, Seeger RC, Shimada H. 
Neuroblastoma of undifferentiated subtype, prognostic significance of prominent nucleolar formation, and MYC/MYCN protein expression: a report from the Children's Oncology Group.

Cancer. 2013; 119: 3718-26.

25. Milla LA, González-Ramírez CN, Palma V. Sonic Hedgehog in cancer stem cells: a novel link with autophagy. Biological research. 2012; 45: 223-30.

26. Souzaki R, Tajiri T, Souzaki M, Kinoshita Y, Tanaka S, Kohashi K, Oda Y, Katano M, Taguchi T. Hedgehog signaling pathway in neuroblastoma differentiation. Journal of pediatric surgery. 2010; 45: 2299-304.

27. Oue T, Yoneda A, Uehara S, Yamanaka H, Fukuzawa M. Increased expression of the hedgehog signaling pathway in pediatric solid malignancies. Journal of pediatric surgery. 2010; 45: 387-92.

28. Amatu A, Sartore-Bianchi A, Siena S. NTRK gene fusions as novel targets of cancer therapy across multiple tumour types. ESMO Open. 2016; 1: e000023.

Table 1 Patients' clinicopathological characteristics in our clinical cohort $(\mathrm{N}=86)$

\begin{tabular}{|c|c|c|c|}
\hline characteristics & Alive $(n=66)$ & Dead $(n=20)$ & Total $(n=86)$ \\
\hline \multicolumn{4}{|c|}{ Age at initial dignosis } \\
\hline$\leq 18 \mathrm{~m}$ & $20(30.3 \%)$ & $2(10 \%)$ & $22(25.6 \%)$ \\
\hline$>18 \mathrm{~m}$ & $46(69.7 \%)$ & $18(90 \%)$ & $64(74.4 \%)$ \\
\hline \multicolumn{4}{|l|}{ Gender } \\
\hline Female & $24(36.4 \%)$ & $5(25 \%)$ & $29(33.7 \%)$ \\
\hline
\end{tabular}




\begin{tabular}{|c|c|c|c|}
\hline Male & $42(63.6 \%)$ & $15(75 \%)$ & $57(66.3 \%)$ \\
\hline \multicolumn{4}{|l|}{ Pathological dignosis } \\
\hline Neuroblastoma & $39(59.1 \%)$ & $13(65 \%)$ & $52(60.5 \%)$ \\
\hline Ganglioneuroblastoma & $23(34.8 \%)$ & $6(30 \%)$ & $29(33.7 \%)$ \\
\hline Ganglioneuroma & $4(6.1 \%)$ & $1(5 \%)$ & $5(5.8 \%)$ \\
\hline \multicolumn{4}{|l|}{ N-MYC status } \\
\hline Amplified & $7(10.6 \%)$ & $14(70 \%)$ & $21(24.4 \%)$ \\
\hline Not amplified & $59(89.4 \%)$ & $6(30 \%)$ & $65(75.6 \%)$ \\
\hline \multicolumn{4}{|l|}{ Losses of 1p } \\
\hline Yes & $3(4.5 \%)$ & $5(25 \%)$ & $8(9.3 \%)$ \\
\hline No & $35(53.1 \%)$ & $6(30 \%)$ & $41(47.7 \%)$ \\
\hline NA & $28(42.4 \%)$ & $9(45 \%)$ & $37(43.0 \%)$ \\
\hline \multicolumn{4}{|l|}{ Losses of 11q } \\
\hline Yes & $4(6.1 \%)$ & $1(5 \%)$ & $5(5.8 \%)$ \\
\hline No & $13(19.7 \%)$ & $3(15 \%)$ & $16(18.6 \%)$ \\
\hline NA & $49(74.2 \%)$ & $16(80 \%)$ & $65(75.6 \%)$ \\
\hline \multicolumn{4}{|l|}{ INSS stage } \\
\hline 1 & $15(22.7 \%)$ & $0(0 \%)$ & $15(17.4 \%)$ \\
\hline 2 & $14(21.2 \%)$ & $0(0 \%)$ & $14(16.3 \%)$ \\
\hline 3 & $10(15.2 \%)$ & $2(10 \%)$ & $12(14.0 \%)$ \\
\hline 4 & $25(37.9 \%)$ & $18(90 \%)$ & $43(50.0 \%)$ \\
\hline $4 s$ & $2(3.0 \%)$ & $0(0 \%)$ & $2(2.3 \%)$ \\
\hline
\end{tabular}


543 Table 2. Basic information of four microarray datasets from GEO database

\begin{tabular}{llll}
\hline Dataset ID & Platform & Sample & Total number \\
\hline GSE16476 & GPL570 & NB & 88 \\
\hline GSE49710 & GPL16876 & NB & 498 \\
GSE73517 & GPL16876 & NB & 105 \\
GSE96631 & GPL22166 & NB; AM; AC; ENCC & 75 (64 NB; 4 AM; 3 \\
& and & & AC; 4 ENCC) \\
& GPL22167 & & \\
\hline
\end{tabular}

544 Abbreviation: AC, adrenal cortex; AM, adrenal marrow; ENCC, embryonic neural 545 crest cells; GEO, Gene Expression Omnibus database; NB, neuroblastoma;

Table 3. Correlation between NTRK1 expression and clinical parameters in 3 GEO datasets

\begin{tabular}{|c|c|c|c|c|c|c|c|c|c|}
\hline \multirow[b]{2}{*}{ variables } & \multicolumn{2}{|l|}{ GSE16476 } & \multirow[b]{2}{*}{$P$} & \multicolumn{2}{|l|}{ GSE49710 } & & \multicolumn{2}{|l|}{ GSE73517 } & \\
\hline & $N T R K I^{\text {low }}$ & $N T R K I^{\text {high }}$ & & $N T R K 1^{\text {low }}$ & $N T R K l^{\text {high }}$ & $P$ & $N T R K 1^{\text {low }}$ & $N T R K I^{\text {high }}$ & $P$ \\
\hline & $(\mathrm{n}=44)$ & $(n=44)$ & & $(\mathrm{n}=\mathbf{2 4 8})$ & $(\mathrm{n}=250)$ & & $(\mathrm{n}=\mathbf{2 4 8})$ & $(\mathbf{n}=\mathbf{2 5 0})$ & \\
\hline
\end{tabular}




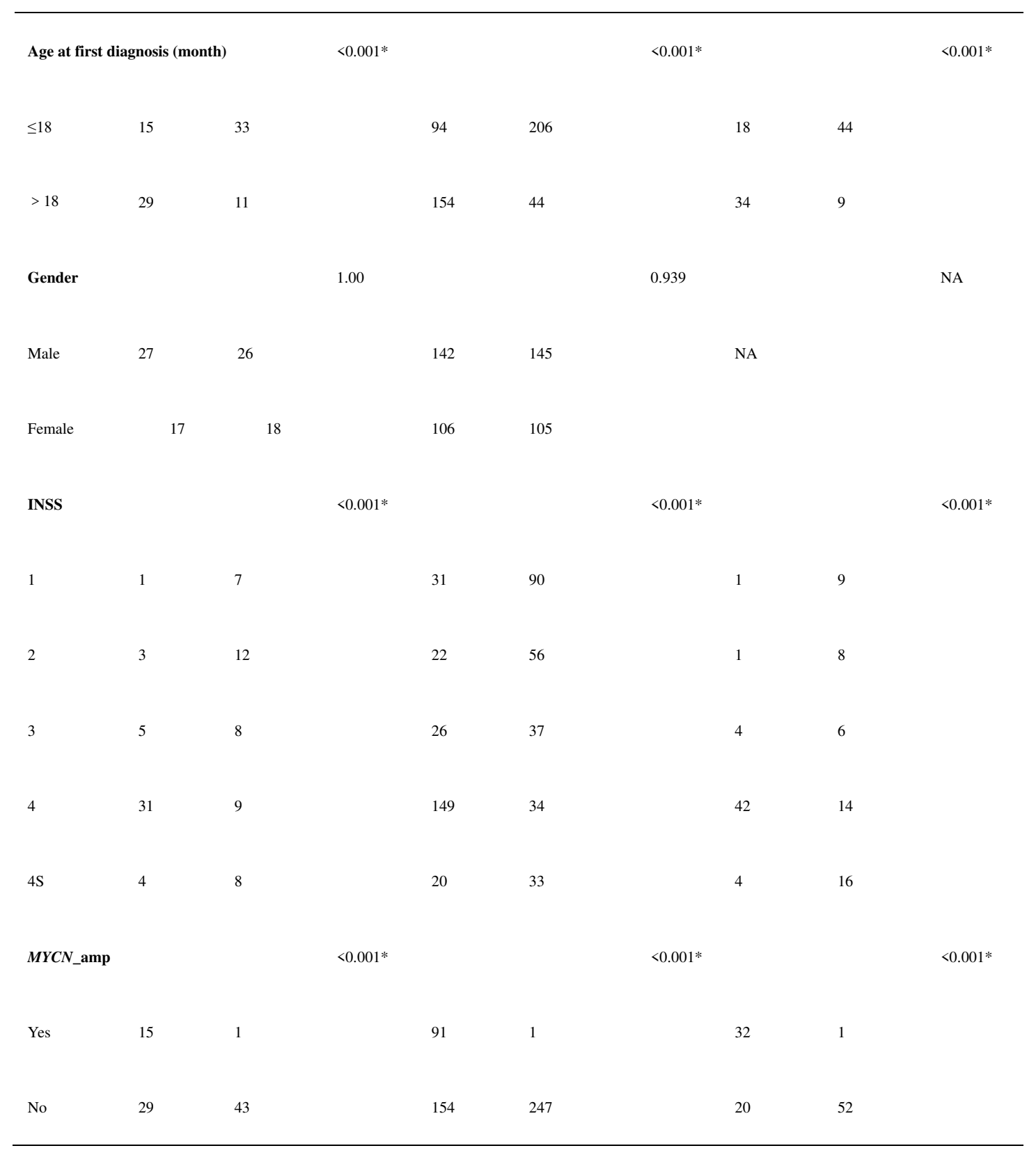

$551 \quad$ Note: * indicates $\mathrm{P}$ value $<0.05$

552 GSE73517 datasets do not provide information about gender of patients.

553 Abbreviation: $M Y C N \_$amp indicates $M Y C N$ gene amplification; NA, not available. 
Table4. Statistics of DEGs from three microarray databasets

\begin{tabular}{llll}
\hline Dataset ID & Upregulated genes & Downregulated genes & Total number \\
\hline GSE16476 & 210 & 197 & 407 \\
GSE49710 & 769 & 64 & 833 \\
GSE73517 & 1839 & 139 & 1978 \\
\hline
\end{tabular}

Table5. Significantly enriched GO terms of DEGs

\begin{tabular}{lllll}
\hline Category & Term & Description & Gene Count & P-value \\
\hline BP_Term & GO:0043547 & positive regulation of GTPase activity & 10 & 0.034343373 \\
BP_Term & GO:0006915 & apoptotic process & 10 & 0.035005858 \\
CC_Term & GO:0005886 & plasma membrane & 58 & $1.54 \mathrm{E}-06$ \\
CC_Term & GO:0005887 & integral component of plasma membrane & 25 & \\
& & & & $2.11 \mathrm{E}-04$ \\
MF_Term & GO:0005509 & calcium ion binding & 16 & $4.13 \mathrm{E}-04$ \\
\hline
\end{tabular}

562

Abbreviations: BP, biological process; CC, cellular component; DEG, differentially expressed gene; GO, Gene Ontology; MF, molecular function.

\section{Figure legends:}

Figure 1. Analysis of the correlation between IHC scores of TrkA and clinical parameters in clinical cohort. a, Immunohistochemistrical semiquantitative integration model. Immunohistochemistrical (IHC) score was based on the degree of staining 
570

571

572 samples, showing different staining status. c, Analysis of the correlation between IHC

573 scores of TrkA and clinical parameters in 86 clinical samples, including age of intial

574 diagnosis ( $\leqslant 18$ month; $>18$ month), MYCN status (amplified;not amplified), gender,

575 and INSS stage. d, TrkA score between primary and metastatic tumor tissue of 32 576 cases.

577 NOTE: $* \mathrm{P}<.05, * * \mathrm{P}<.01, * * * \mathrm{P}<.001, * * * * \mathrm{P}<0.001, \quad$ ns, no statistical difference 578 (P>0.05). IHC, immunohistochemistry; NB, neuroblastoma.

579

580

Figure 3. Survival analyses, time-dependent ROC curves and DCA curves for the

591 prognostic model in clinical cohort and GEO cohort. a, Kaplan - Meier overall 
592

593

594

595

596

597

598

601

602

603

604

605

606

607

608

609

610

611

612

613

survival (OS) analyses for NTRK1/TrkA expression in86 clinical NB samples $(\mathrm{P}=0.0001), 88$ patients of GSE16476 $(\mathrm{P}<0.0001)$ and 498 patients of GSE49710 ( $\mathrm{P}<0.0001)$. b-d, Multivariate cox regression analysis of risk factors associated with overall survival (OS) times of clinical samples, GSE16476 and GSE49710 respectively. e, Survival prediction power of the NTRK1/TrkA signature was determined by ROC analysis. The AUC values of clinical samples, GSE16476 and GSE49710 were calculated separately from the ROC curve. f, Decision Curve Analysis of clinical samples, GSE16476 and GSE49710.

Note: $M Y C N$-amp, $M Y C N$ gene amplification; CI, confidence interval.

Figure 4. Nomogram to predict the probabilities of 1,2 and 3-year survival probability. Points are assigned for pathological diagnosis, TrkA score, Tumor INSS stage, age of initial diagnosis and $M Y C N$ status by drawing a line upward from the corresponding values to the "Points" line. Draw an upward vertical line to the "Points" bar to calculate points. Based on the sum, draw a downward vertical line from the "Total Points" line to calculate 1, 2 and 3-year survival probability. Internal validation using the bootstrap method showed that the $\mathrm{C}$-index for the model was 0.95 .

Figure 5. Detection for NTRK1 gene fusion. a, Fluorescence in-situ hybridization (FISH) of NTRK1 "break-apart" probe. Separation signal was detected within $15 \%$ of tumor cells which indicated there was no fusion in NTRK1. b, qRT-PCR amplification 
614 plot of positive control. The CT value of HEX (VIC) signal for internal control was $61512.5(\leqslant 20)$, and CT value of FAM signal for target gene was $13.43(\leqslant 25)$. c, 616 qRT-PCR amplification plot of clinical sample. The CT value of HEX (VIC) signal 617 was $12.27(\leqslant 20)$, and CT value of FAM signal for target gene was below detection 618 limitation.

Figure 6. Identification of differentially expressed genes (DEGs) between NB-NTRKI $1^{\text {high }}$ and NB-NTRK1 $1^{\text {low }}$ groups in three datasets (GSE16476, GSE49710

622

623

629

630

631

632

633 and GSE73517). a, Volcano plot showing the DEGs in three datasets. The red points represent upregulated genes screened based on the criteria of $\mathrm{P}<0.05$ and $\log \mathrm{FC} \geqslant$ 1.The blue points represent downregulation of the expression of genes screened based on the criteria of $\mathrm{P}<0.05$ and $\operatorname{logFC} \leqslant-1$. The black points represent genes with no significant difference. b, Venn diagram of upregulated and downregulated DEGs common to all three GEO datasets. c, PPI network constructed with the DEGs, green nodes are upregulated genes, and yellow nodes are downregulated genes.

Figure 7. Gene set variation analysis (GSVA) for the NTRK1 in three GEO datasets. a-c, The heatmaps of differentially expressed pathways for NTRK1 through the calculation of GSVA in GSE16476, GSE49710, GSE373517 respectively. d, Venn diagram of pathways to three GEO datasets. 


\section{Figures}

a
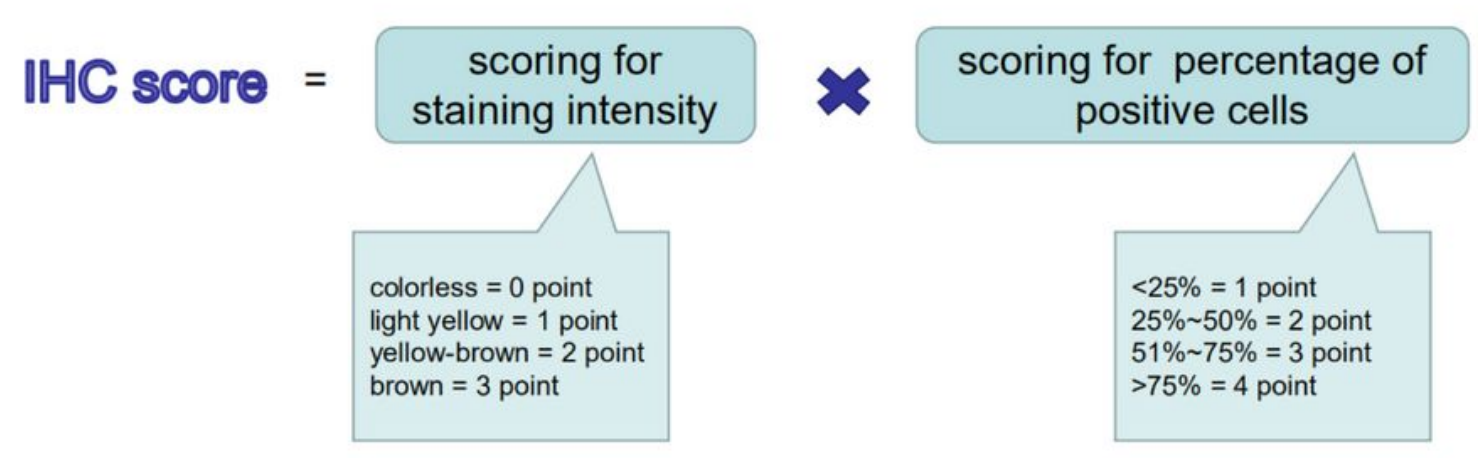

b
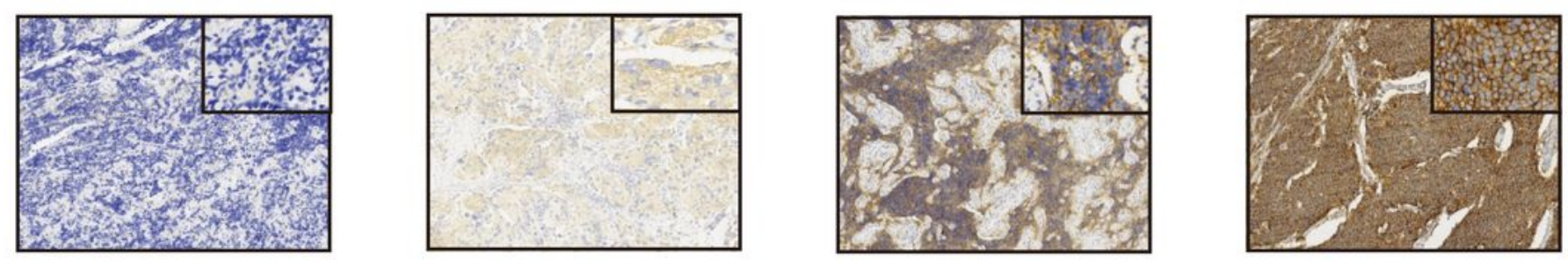

C
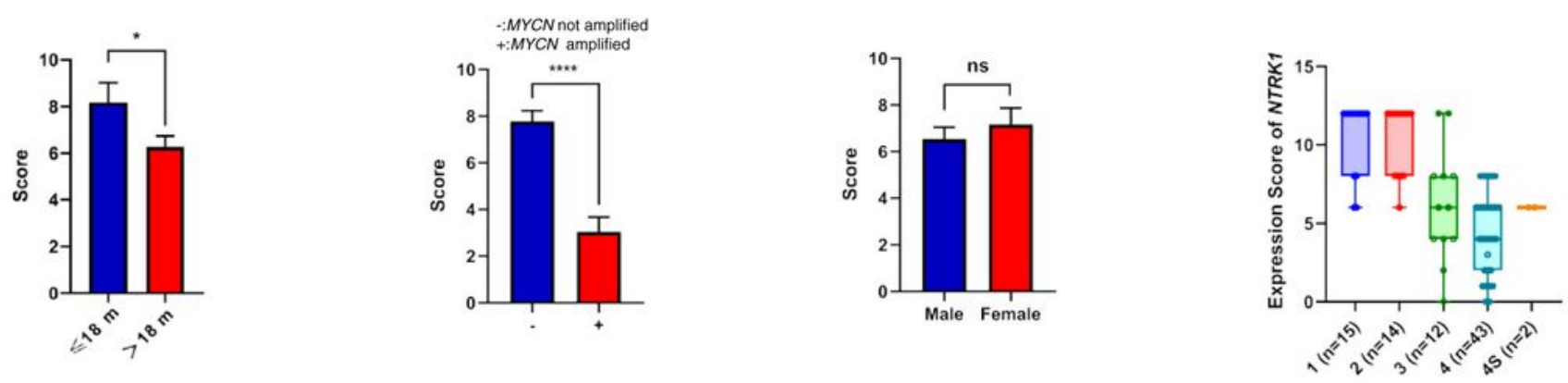

d

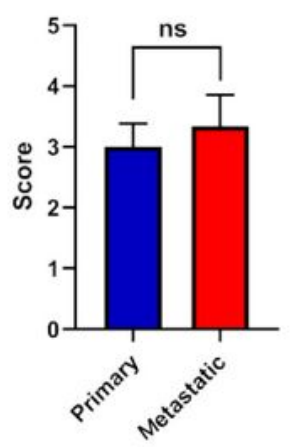

\section{Figure 1}

Analysis of the correlation between IHC scores of TrkA and clinical parameters in clinical cohort. a, Immunohistochemistrical semiquantitative integration model. Immunohistochemistrical (IHC) score was based on the degree of staining intensity and percentage. The final IHC score $=$ staining intensity score $\times$ 
positive rate score. $\mathrm{b}$, TrkA was stained by anit-TrkA by IHC technique in the $86 \mathrm{NB}$ clinical samples, showing different staining status. c, Analysis of the correlation between IHC scores of TrkA and clinical parameters in 86 clinical samples, including age of intial diagnosis ( $\leq 18$ month; 818 month), MYCN status (amplified;not amplified), gender, and INSS stage. $d$, TrkA score between primary and metastatic tumor tissue of 32 cases. NOTE: ${ }^{*} P<.05,{ }^{*} \mathrm{P}<.01,{ }^{\star} * \star \mathrm{P}<.001, \star \star \star \star \mathrm{P} \otimes 0.001, \mathrm{~ns}$, no statistical difference $(P>0.05)$. IHC, immunohistochemistry; NB, neuroblastoma.

a
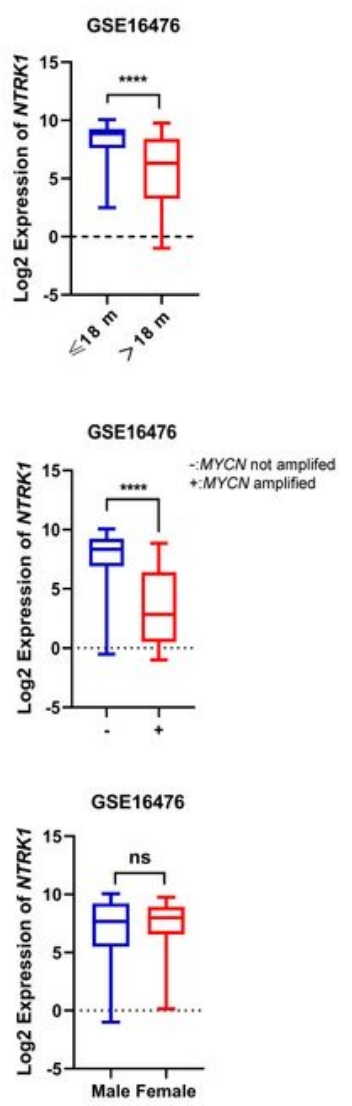

d

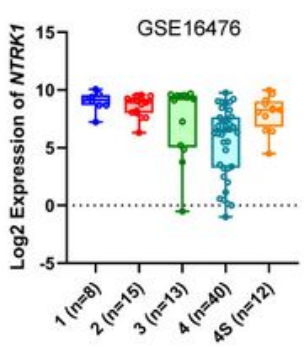

e

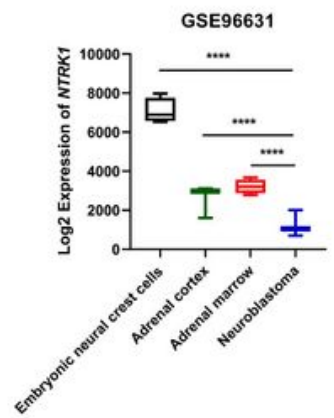

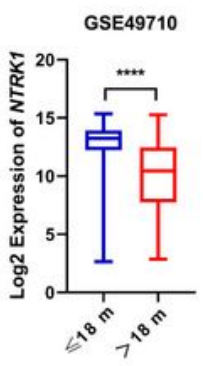
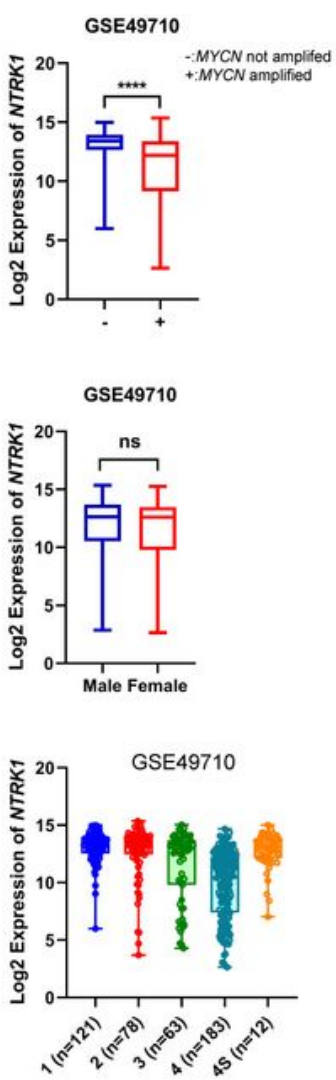
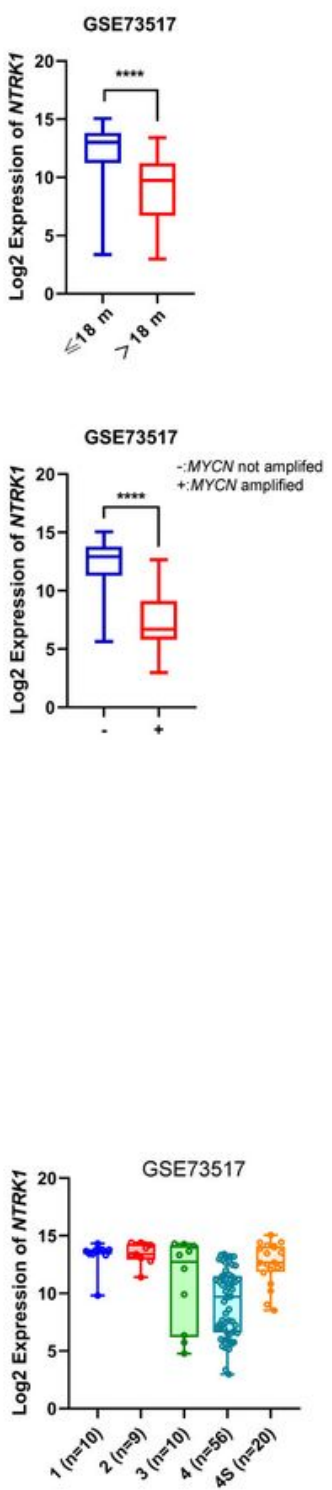

Figure 2 
Associations between NTRK1 expression and clinical paraments in three public datasets (GSE16476, GSE49710 and GSE73517). a, NTRK1 expression and age of initial diagnosis( $\leq 18$ month;®18 month). b, NTRK1 expression and MYCN status (amplified; not amplified). c, NTRK1 expression and gender. d, NTRK1 expression among different INSS stage. e, NTRK1 mRNA expression in NB $(n=64) \rrbracket$ embryonic neural crest cells $(n=4)$ Dadrenal cortex $(n=3)$ and adrenal marrow $(n=4)$ TrkA expression in GSE96631.

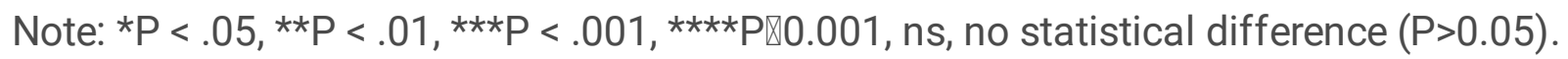

a

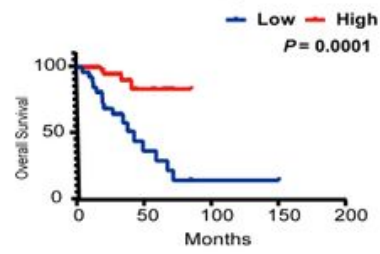

GSE16476

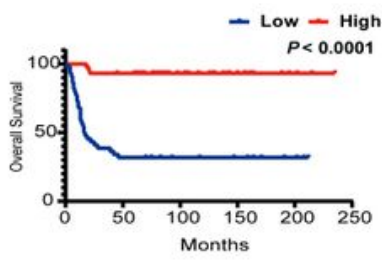

variables P Hazard Ratio [95\% Cl]

age $\quad 0.912$

sex $\quad 0.746$

stage $\quad 0.262$

MYCN-amp $\quad 0.027$

TrkA score $\quad 0.035$

C

$\begin{array}{lc}\text { variables } & P \\ \text { age } & <0.001 \\ \text { sex } & 0.342 \\ \text { stage } & 0.485 \\ \text { MYCN-amp } & 0.997 \\ \text { NTRK1 } & 0.001\end{array}$

d

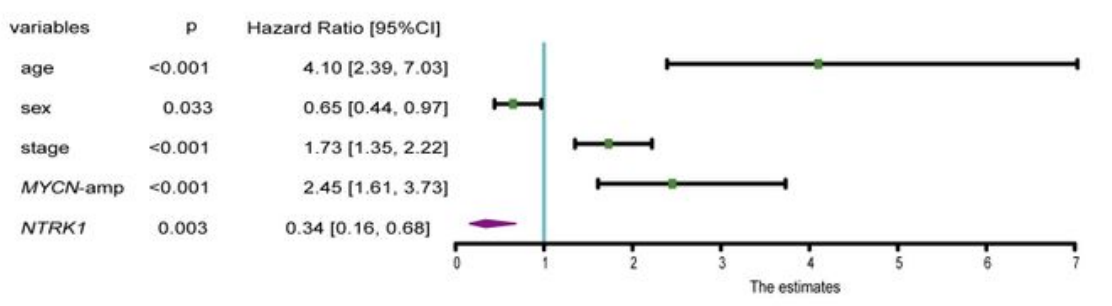

e
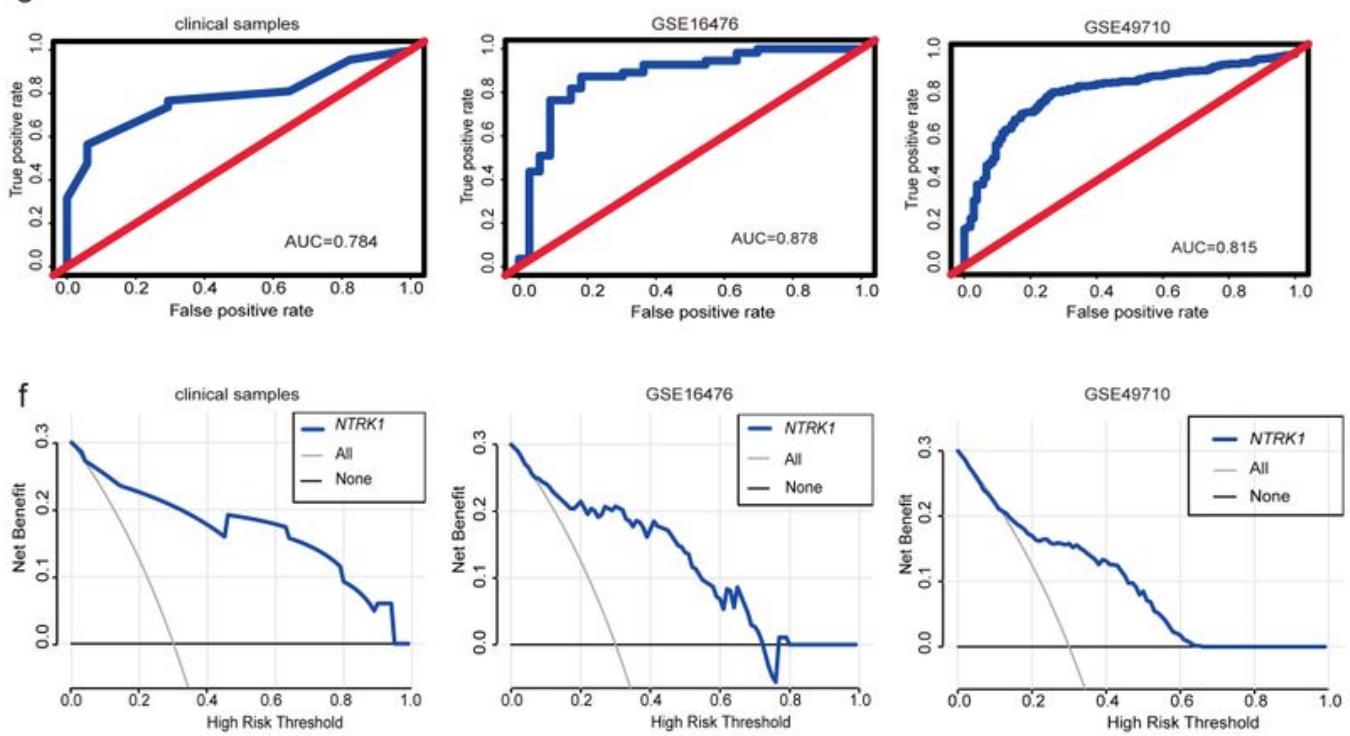

GSE49710
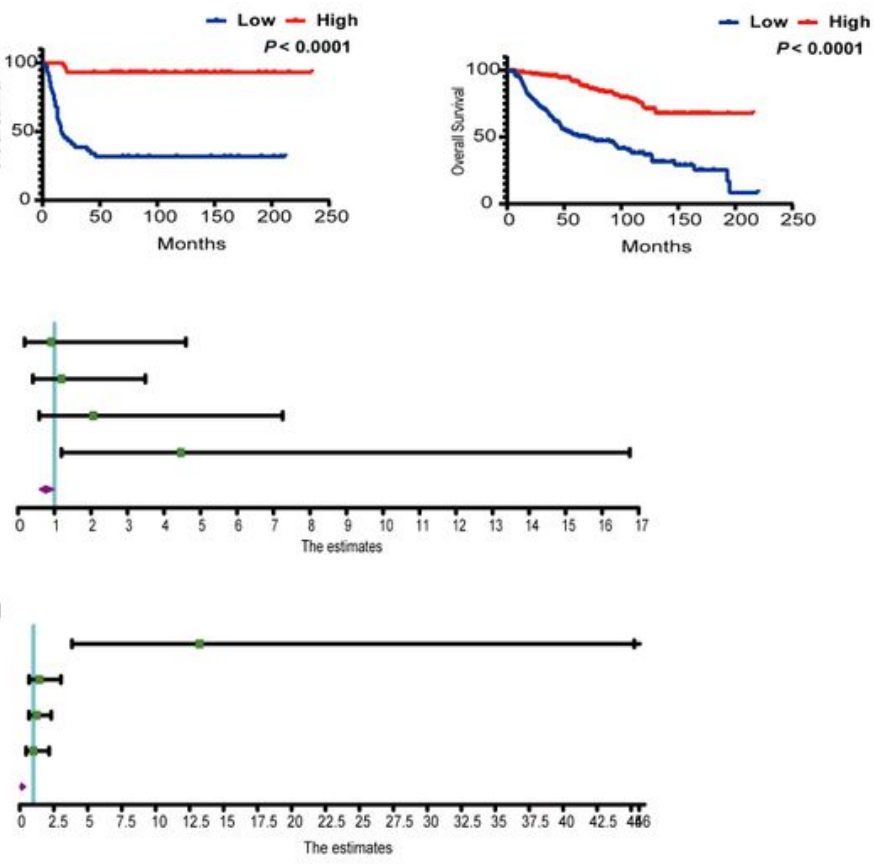

Hazard Ratio [95\%Cl]

$3.23[3.84,45.64]$

$1.44[0.68,3.03]$

. $25[0.67,2.31]$

$0.11[0.03,0.40]$

The estimates

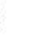


Survival analyses, time-dependent ROC curves and DCA curves for the prognostic model in clinical cohort and GEO cohort. a, Kaplan-Meier overall survival (OS) analyses for NTRK1/TrkA expression in86 clinical NB samples $(P=0.0001), 88$ patients of GSE16476 『Pख0.0001『and 498 patients of GSE49710 (Pख0.0001). b-d, Multivariate cox regression analysis of risk factors associated with overall survival (OS) times of clinical samples, GSE16476 and GSE49710 respectively. e, Survival prediction power of the NTRK1/TrkA signature was determined by ROC analysis. The AUC values of clinical samples, GSE16476 and GSE49710 were calculated separately from the ROC curve. f, Decision Curve Analysis of clinical samples, GSE16476 and GSE49710. Note: MYCN-amp, MYCN gene amplification; $\mathrm{Cl}$, confidence interval.

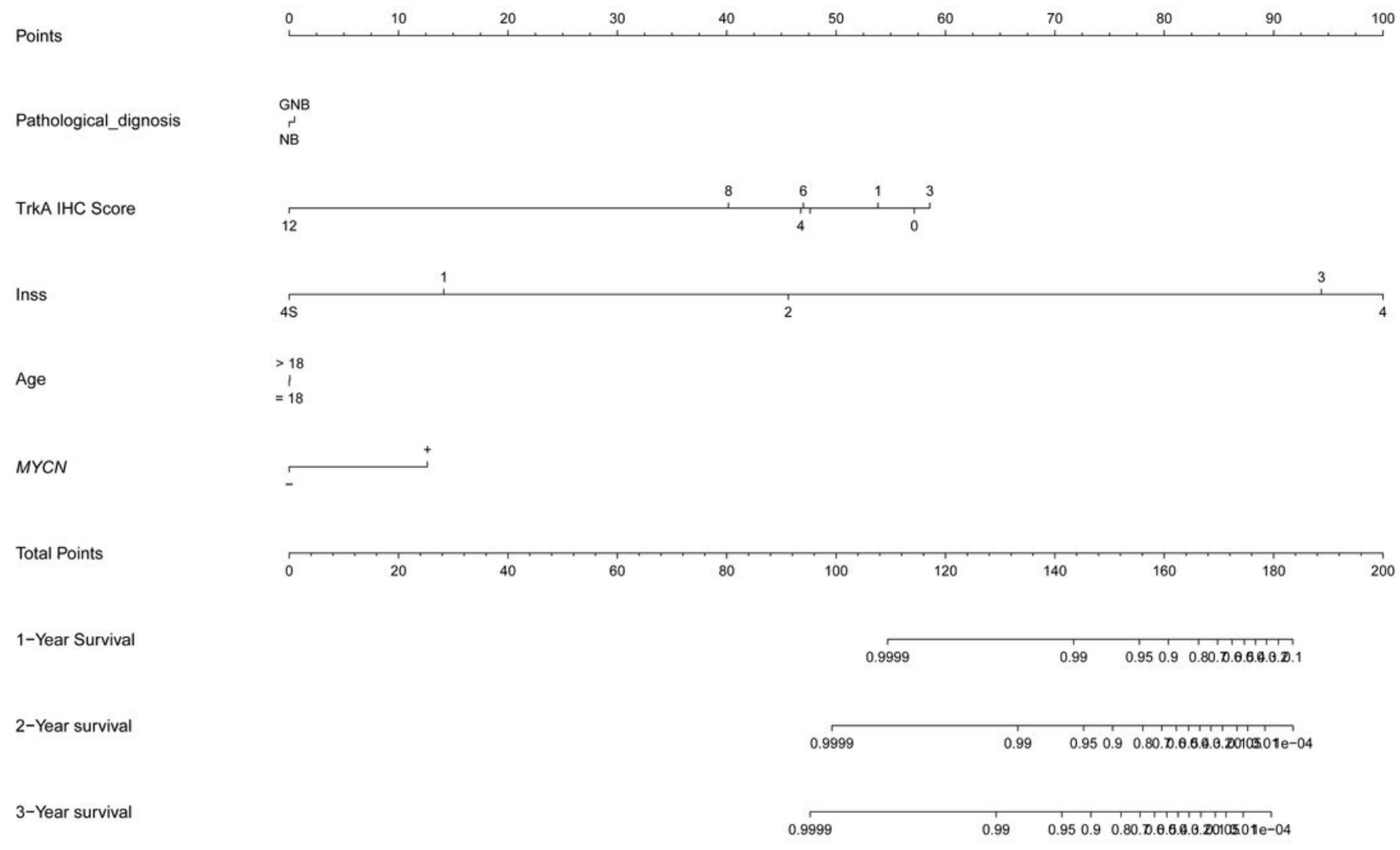

\section{Figure 4}

Nomogram to predict the probabilities of 1,2 and 3-year survival probability. Points are assigned for pathological diagnosis, TrkA score, Tumor INSS stage, age of initial diagnosis and MYCN status by drawing a line upward from the corresponding values to the "Points" line. Draw an upward vertical line to the "Points" bar to calculate points. Based on the sum, draw a downward vertical line from the "Total Points" line to calculate 1, 2 and 3-year survival probability. Internal validation using the bootstrap method showed that the C-index for the model was 0.95 . 
a
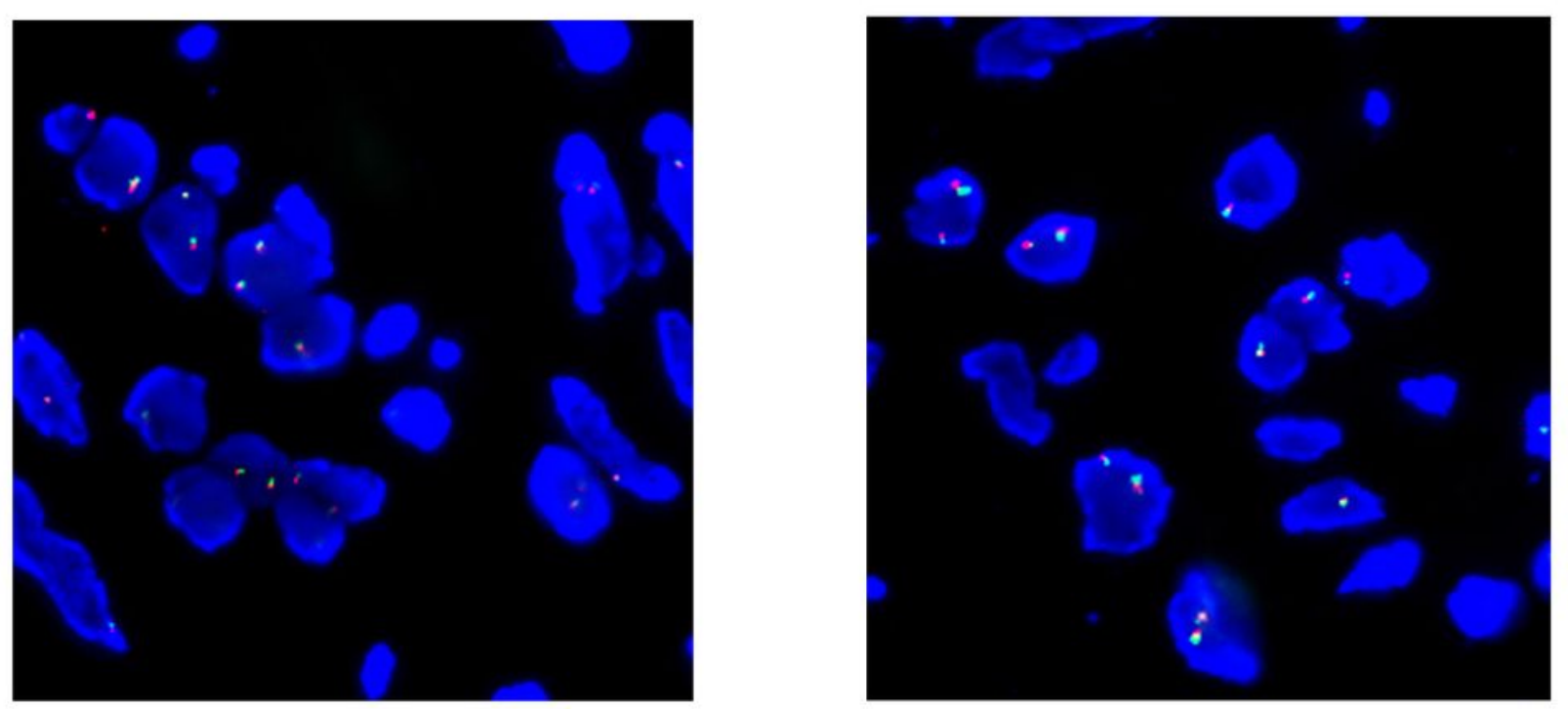

b
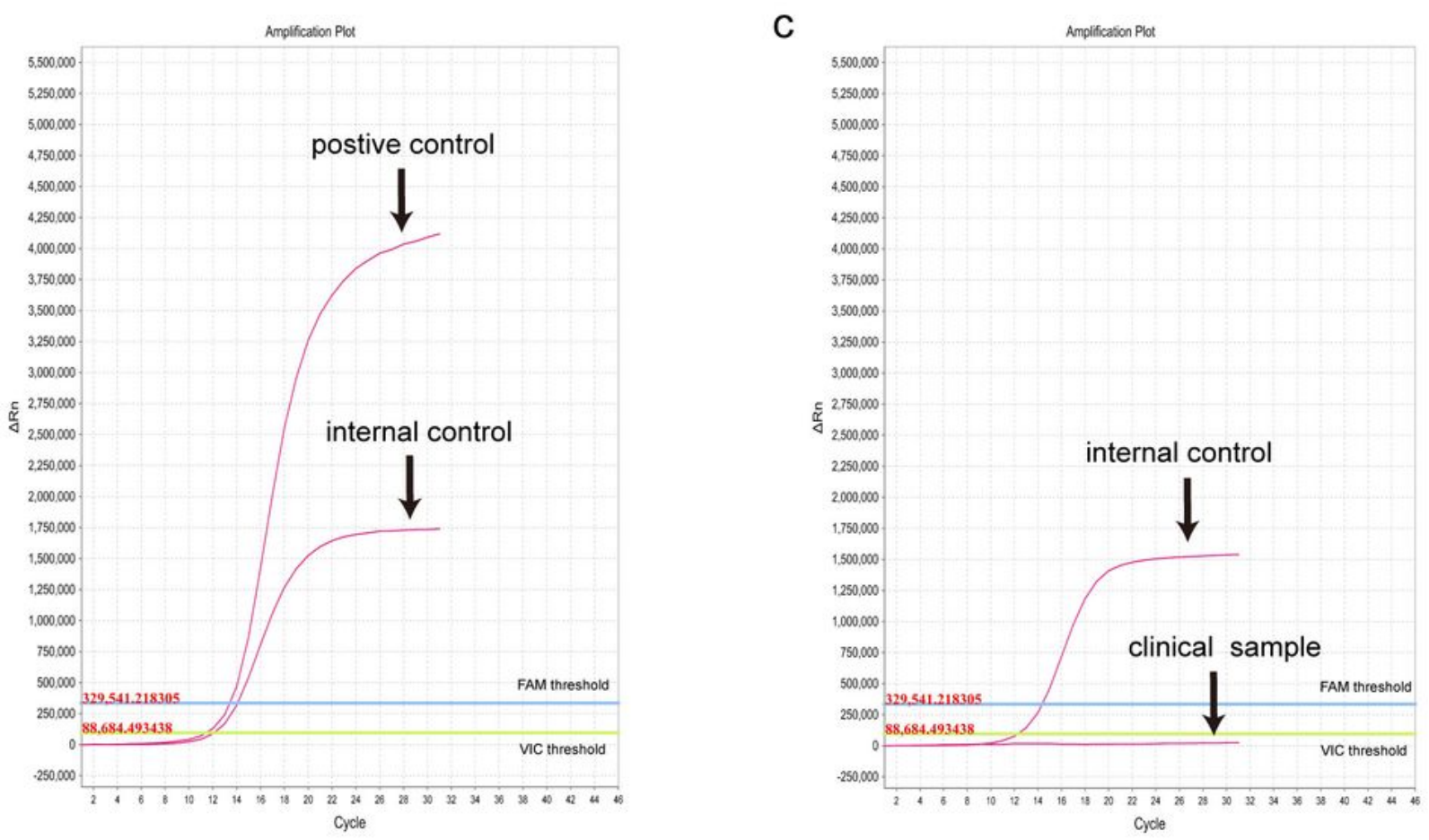

\section{Figure 5}

Detection for NTRK1 gene fusion. a, Fluorescence in-situ hybridization (FISH) of NTRK1 "break-apart" probe. Separation signal was detected within $15 \%$ of tumor cells which indicated there was no fusion in NTRK1. b, qRT-PCR amplification plot of positive control. The CT value of HEX (VIC) signal for internal control was $12.5(\leq 20)$, and CT value of FAM signal for target gene was $13.43(\leq 25)$. C, qRT-PCR 
amplification plot of clinical sample. The CT value of HEX (VIC) signal was $12.27(\leq 20)$, and CT value of FAM signal for target gene was below detection limitation.
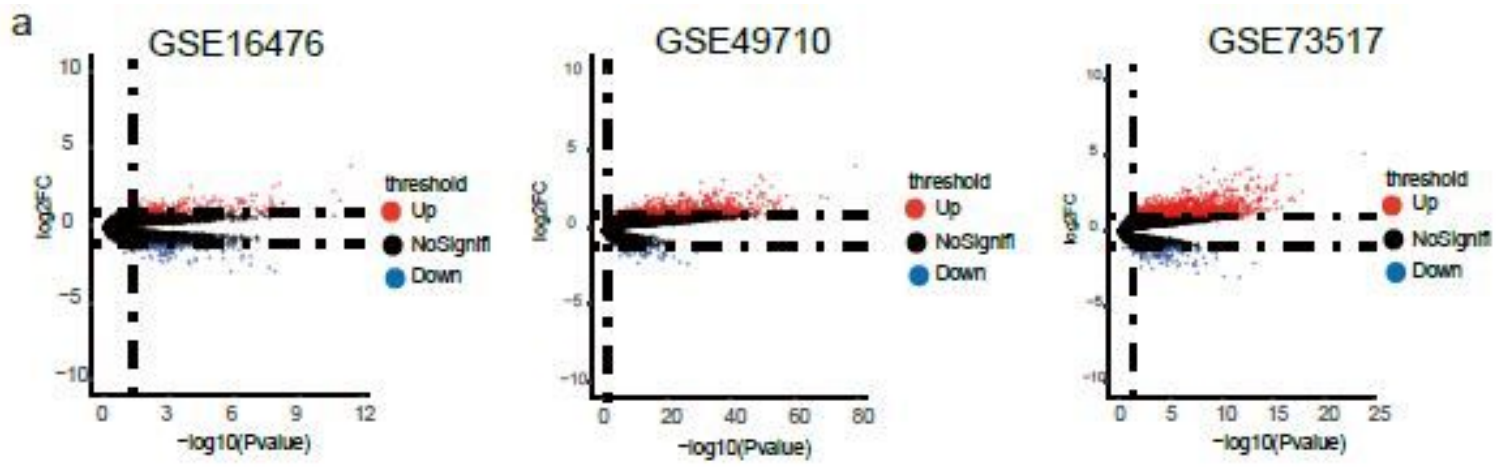

b
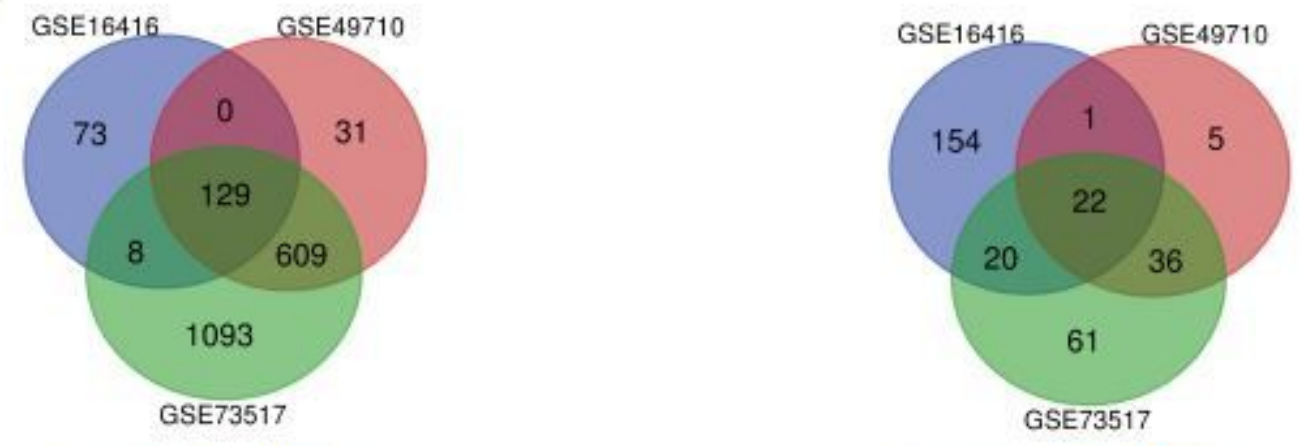

upregulated DEGs

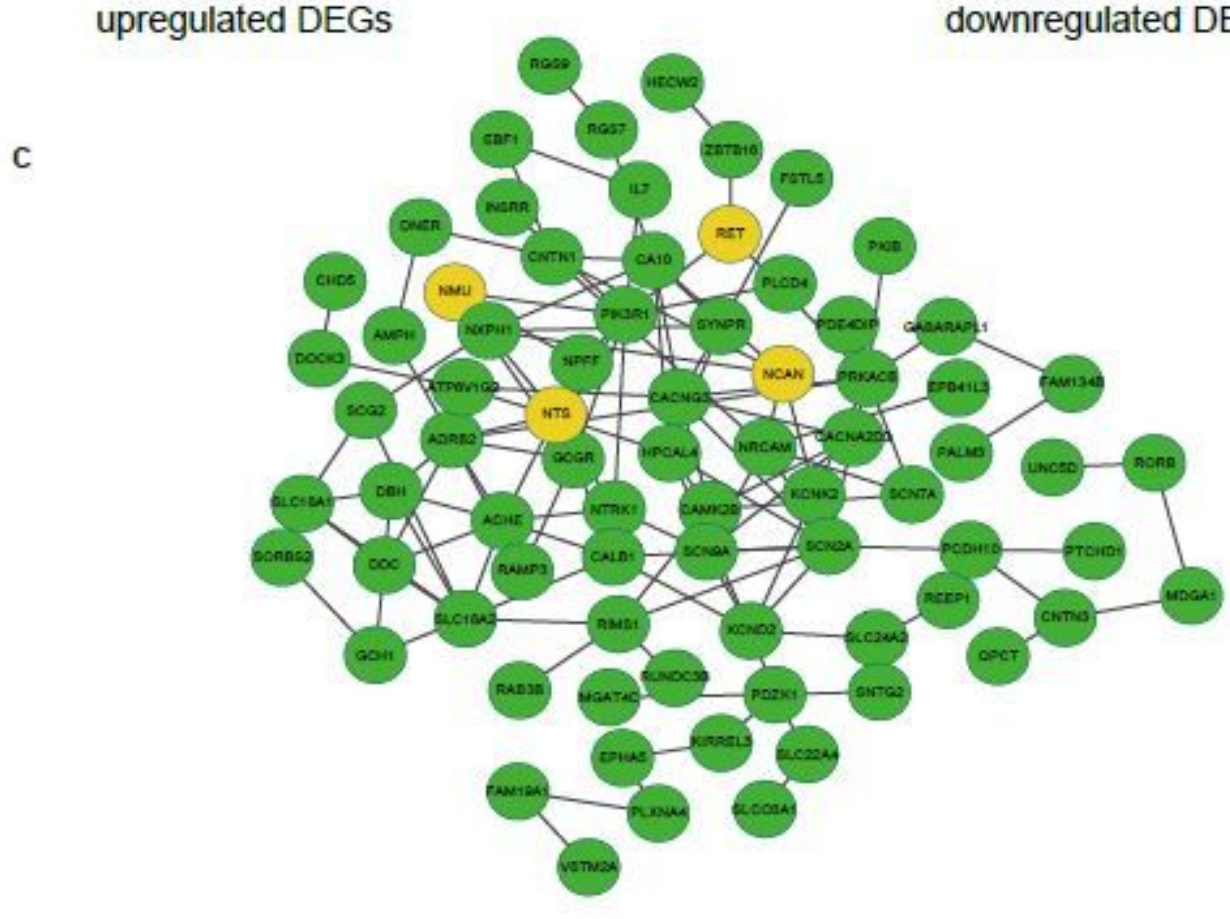

\section{Figure 6}

Identification of differentially expressed genes (DEGs) between NB-NTRK1 high and NB-NTRK1low groups in three datasets (GSE16476, GSE49710 and GSE73517). a, Volcano plot showing the DEGs in three datasets. The red points represent upregulated genes screened based on the criteria of Pख0.05 and $\log \mathrm{FC} \geq 1$. The blue points represent downregulation of the expression of genes screened based on the 
criteria of $\mathrm{P} \otimes 0.05$ and $\log \mathrm{FC} \leq-1$. The black points represent genes with no significant difference. $b, V e n n$ diagram of upregulated and downregulated DEGs common to all three GEO datasets. c, PPI network constructed with the DEGs, green nodes are upregulated genes, and yellow nodes are downregulated genes.

a

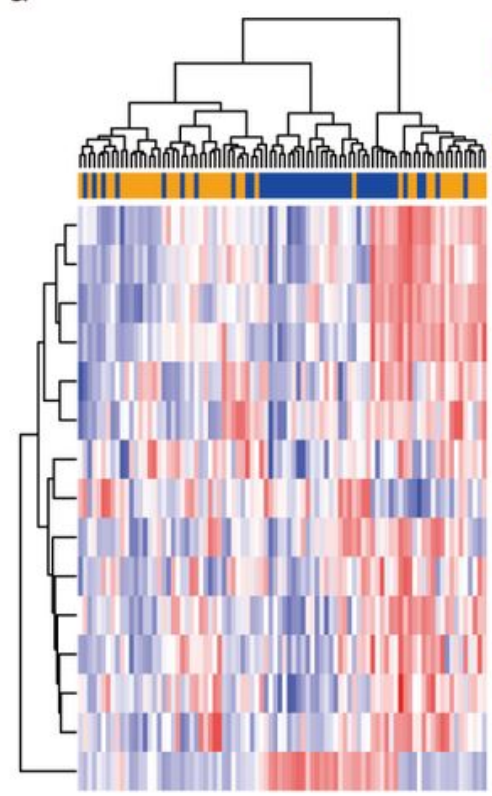

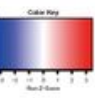

NTRK1 high expression HALLMARK_KRAS_SIGNALING_UP HALLMARK_APOPTOSIS HALLMARK_TGF_BETA_SIGNALING HALLMARK_UV_RESPONSE_DN HALLMARK_APICAL_SURFACE HALLMARK_WNT_BETA_CATENIN_SIGNALING HALLMARK_PANCREAS_BETA_CELLS HALLMARK_SPERMATOGENESIS HALLMARK_CHOLESTEROL_HOMEOSTASIS HALLMARK_PI3K_AKT_MTOR_SIGNALING HALLMARK_PROTEIN_SECRETION HALLMARK_ANDROGEN_RESPONSE HALLMARK_HEME_METABOLISM HALLMARK_ADIPOGENESIS HALLMARK_MYC_TARGETS_V2 b

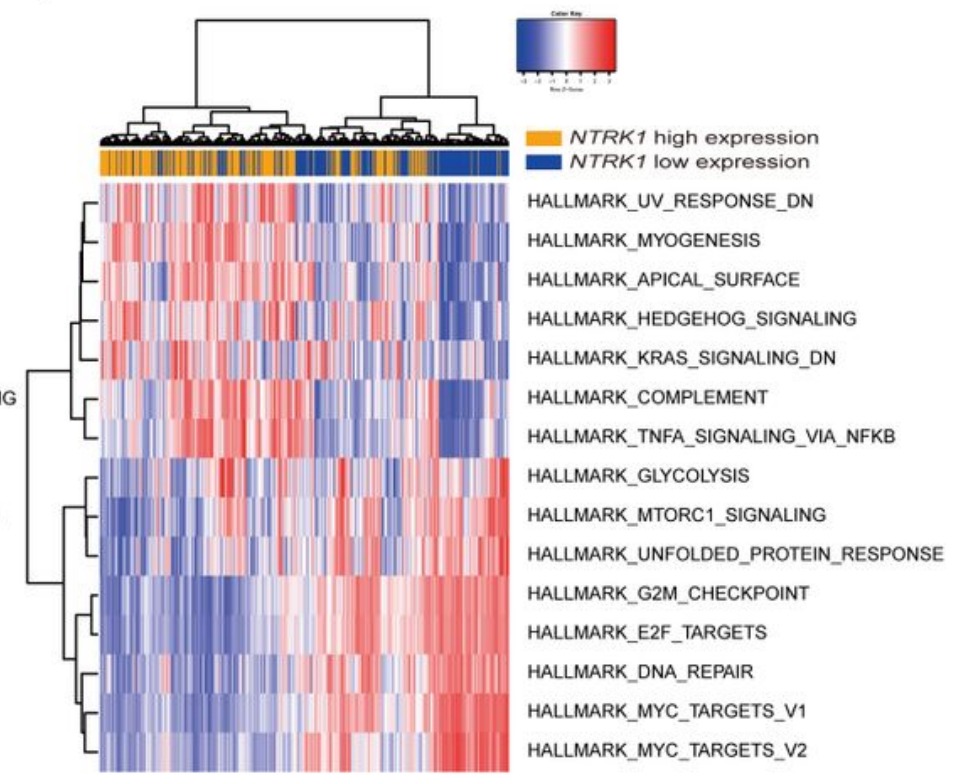

C

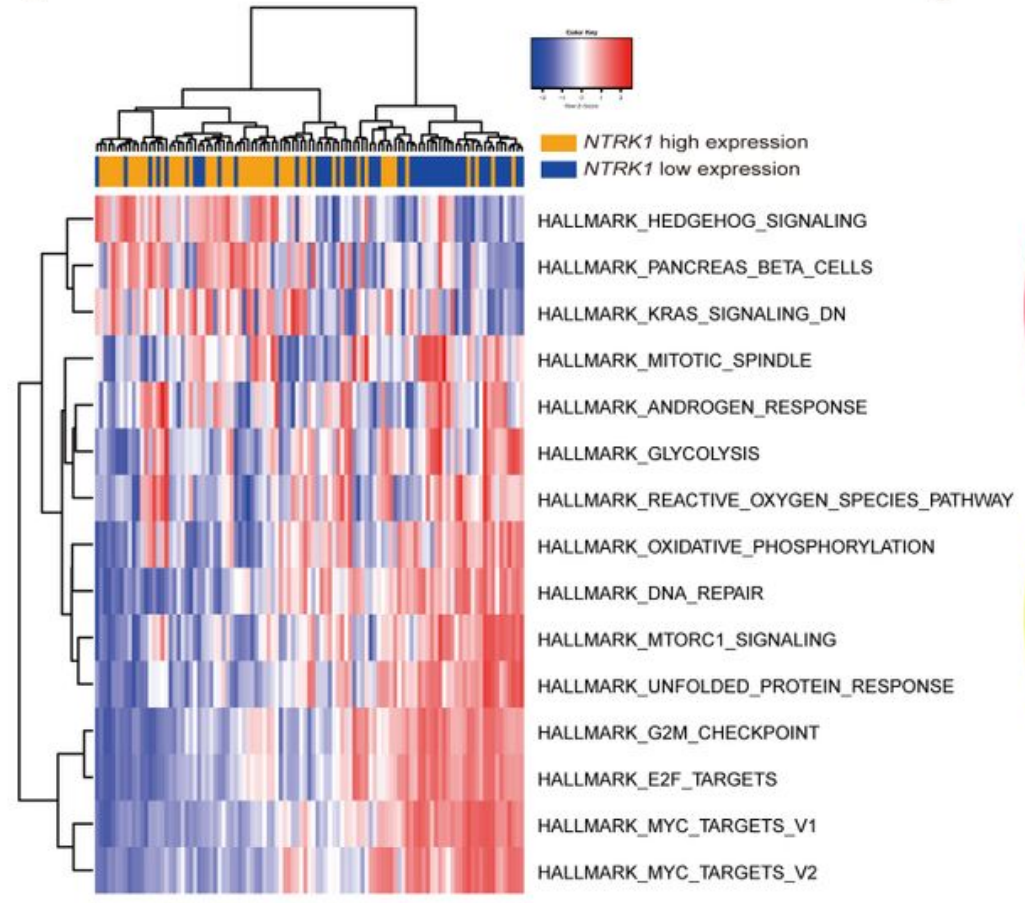

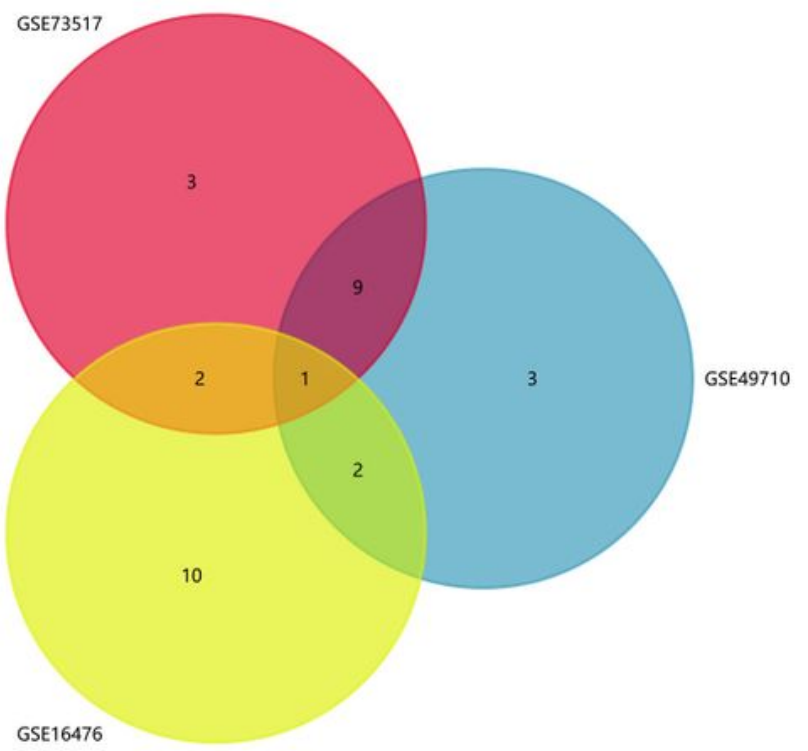

Figure 7 
Gene set variation analysis (GSVA) for the NTRK1 in three GEO datasets a-c, The heatmaps of differentially expressed pathways for NTRK1 through the calculation of GSVA in GSE16476, GSE49710, GSE373517 respectively. d, Venn diagram of pathways to three GEO datasets.

\section{Supplementary Files}

This is a list of supplementary files associated with this preprint. Click to download.

- Tables1.xls

- Tables2.xls

- Tables3.xls

- Tables4.xls

- TableS5.xlsx

- TableS6.xIsx

- TableS7.xlsx

- Tables8.xls

- Tables9.xls 